\title{
FABRICATION OF SUB-MICRON METAL WIRES FOR HIGH-FREQUENCY LITZ WIRE
}

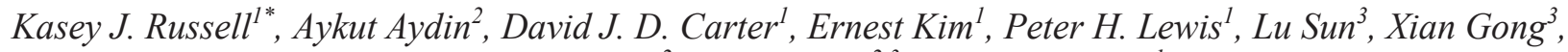 \\ Christina Chang ${ }^{2}$, Roy Gordon ${ }^{2,3}$, and Amy Duwel ${ }^{1}$ \\ ${ }^{1}$ The Charles Stark Draper Laboratory, Inc., Cambridge MA, USA \\ ${ }^{2}$ Department of Chemistry and Chemical Biology, Harvard University, Cambridge MA, USA \\ ${ }^{3}$ John A. Paulson School of Engineering and Applied Sciences, Harvard University, Cambridge MA, USA
}

\begin{abstract}
At $\mathrm{GHz}$ frequencies and above, ohmic losses associated with $\mu \mathrm{m}$-scale skin depth limit the maximum quality factor, $\mathrm{Q}$, that can be achieved in inductors. We demonstrate fabrication of sub-micron diameter wires over centimeter-scale lengths from a wide variety of materials including copper, gold, and platinum/palladium. By depositing metal and dielectric materials onto a high-strength nanofiber scaffold, we have made conducting wires, insulated conducting wires, and coaxial multilayer structures. Additionally, we have demonstrated the ability to manipulate sub-micron wires into ordered, twisted bundles while maintaining electrical properties.
\end{abstract}

\section{INTRODUCTION}

In radio-frequency and microwave devices, many sophisticated fabrication procedures have been developed to improve the quality factor, Q, of MEMS inductors by reducing coupling to the substrate[1]-[3]. Further improvement in Q is limited by skin effect in the metal and its associated increase in ohmic losses[3]. These ohmic losses could be reduced by using a bundle of thin, hierarchically twisted wires (i.e. litz wire), but it is difficult to achieve the necessary topology using conventional 2D MEMS processing[4], [5]. Traditional litz wire fabrication approaches can achieve the necessary topology but cannot scale to $\mathrm{GHz}$ frequencies for two reasons: first, the necessary wire diameter $(\sim 1$ micron and smaller) is an order of magnitude below the limit of conventional wire drawing, and second, conventional manipulation approaches are not compatible with such fragile wire.

Here we demonstrate methods to overcome both wire fabrication and wire manipulation limitations of conventional approaches. We first demonstrate a method to fabricate sub-micron diameter wires over centimeter-scale lengths. Rather than form fine wire by reducing the diameter of larger wire, as is done in traditional drawing, our approach leverages MEMS fabrication techniques to deposit the desired wire material onto a high-strength nanofiber scaffold. Using this method, we have made conducting wires, insulated conducting wires, and coaxial multilayer structures. We then demonstrate the ability to manipulate sub-micron wires into ordered, twisted bundles while maintaining electrical properties. Using fluid drag, we are able to apply sufficient tension to the wires to enable manipulation and plastic deformation without risk of fracture.

The paper is organized as follows: In the Theory section, we provide a brief overview of the skin effect and its implications. In the Wire Fabrication section, we show preparation of individual centimeter-length nanofiber scaffolds formed via electrospinning from poly(m-phenylene isophthalamide). By varying process parameters, we are able to achieve uniform individual nanofibers of diameters ranging from $300 \mathrm{~nm}$ to $600 \mathrm{~nm}$. We then show methods to fixture arrays of nanofibers to enable deposition through a variety of processes including physical vapor deposition, chemical vapor deposition, and atomic layer deposition. Finally, in the Wire
Manipulation section, we show how hydrodynamic flow fields can be utilized to tension and maintain absolute position of individual wires, allowing the manipulation of insulated, metal-coated nanofibers into centimeter-long bundles of twisted wires. Further, we demonstrate that the process can be continued hierarchically, forming twisted bundles of twisted bundles (as in conventional litz wire). Finally, we show how these techniques provide a path to nanoscale, $\mathrm{GHz}$ frequency litz wire.

\section{THEORY}

Skin effect and the related proximity effect have been known since the 1800s. As described by Maxwell's equations, a timevarying current in a conductor induces time-varying magnetic fields, which in turn induce time-varying electric fields and currents that can oppose the flow of current through the conductor. At sufficiently high frequencies, the current through the core of a conductor is suppressed, and power is conducted primarily in an outer layer whose thickness, called the skin depth $\delta$, is given by the equation

$$
\delta=(\rho / \pi f \mu)^{1 / 2},
$$

where $\rho$ and $\mu$ are the resistivity and magnetic permeability of the conductor, respectively, and $f$ is the frequency of the time-varying current.

As illustrated in Fig. 1, skin effect greatly reduces the fraction of the wire cross-section that carries current. Finite element model (FEM) simulations (COMSOL) enable quantitative analysis (Fig. 1(a-b)). For a wire with $r>>\delta$, where $r$ is the radius of the wire, a simple estimate can be obtained by assuming the current flows only in an outer layer of thickness $\delta$. This approximation becomes less accurate for small $r$, but nevertheless provides a way to quickly estimate what wire diameters are appropriate for a given frequency (Fig. 1(c)).

Confining the current to a thin surface layer at higher frequencies leads to higher resistive losses at higher frequencies. The increase in resistance versus frequency can be characterized by the 'resistance ratio' $R_{R}=R_{A C} / R_{D C}$, where $R_{A C}$ is the high-frequency resistance and $R_{D C}$ is the zero-frequency resistance. At the $5 \mathrm{G}$ band near $28 \mathrm{GHz}$, for example, a 58 AWG copper wire would have $R_{R}$ near 10 and a typical 1 mil diameter bond wire would have $R_{R} \sim 20$ (Fig. 1(c)). By comparison, a single copper wire fabricated by our method would have $R_{R} \sim 1$.

Decreasing wire diameter can reduce $R_{R}$, but it obviously increases the absolute resistance of the wire. A solid copper wire of $\sim 1 \mu \mathrm{m}$ diameter has a resistance per unit length greater than 200 $\Omega / \mathrm{cm}$. In a real application, many such wires would be individually insulated and connected electrically in parallel to yield a lowimpedance bundle. However, if the wires are simply packed in a bundle, with wires at the periphery remaining at the periphery and wires at the center remaining at the center, then proximity effect would suppress conduction in the wires located in the middle of the bundle. Proximity effect and skin effect are analogous effects that occur in stranded or continuous conductors. To mitigate proximity effect, the wires can be braided or twisted together hierarchically 
such that wires 'take turns' at the center of the bundle, an arrangement known as 'litz wire'. Typically, the optimal geometry and strand diameter will depend on application, and extensive design guides have been established to aid in analysis[6].

(a)

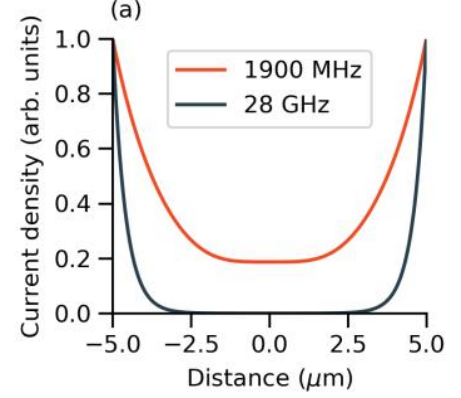

(c)

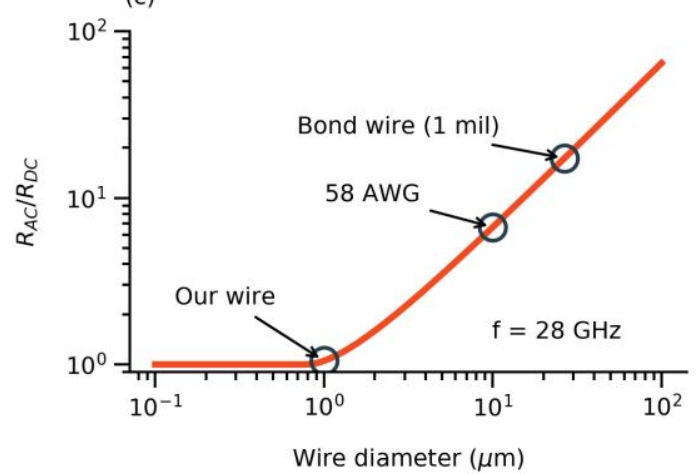

Figure 1:Illustration and ramifications of skin effect. (a), (b), Finite element model simulations of current density in a commercial cylindrical copper wire (58 AWG, $\sim 10 \mu m$ diameter). (a), Normalized current density as a function of position on a section line through the center of a wire at frequencies of $1900 \mathrm{MHz}$ (a common $4 \mathrm{G}$ wireless band) and $28 \mathrm{GHz}$ (the lowest-frequency $5 \mathrm{G}$ Upper Microwave Flexible Use service band). (b), Normalized current density at $28 \mathrm{GHz}$. (c), Analytical estimate of resistance ratio $R_{R}=R_{A C} / R_{D C}$ as a function of wire diameter at a frequency of $28 \mathrm{GHz}$.

As can be seen in Fig. 1, skin effect leads to significant losses at $\mathrm{GHz}$ frequencies, even in the smallest available commercial wire. To enable development of low-loss conductors for future highfrequency technologies, such as communication on the $5 \mathrm{G}$ band at $28 \mathrm{GHz}$, a new technology for litz wire is needed that is compatible with individual strands of diameter near $\sim 1 \mu \mathrm{m}$.

\section{WIRE FABRICATION}

Our wire fabrication process consists of conformal deposition of metallic and dielectric layers onto a nanofiber scaffold core (Fig. 2). For the nanofiber core, the polymer poly(m-phenylene isophthalamide) (PMIA, trade name Nomex) was selected for its high strength as well as its ability to withstand process temperatures up to $350{ }^{\circ} \mathrm{C}$ (Sigma Aldrich). PMIA was dissolved into a solution of $\mathrm{CaCl}_{2}$, loaded into a syringe in a commercial electrospinning apparatus (EC-CLI, IME Technologies), and accelerated toward a rotating spoked-drum collector via a high-voltage bias (typically 6$12 \mathrm{kV}$ ). Electrospinning was performed in an environmentallycontrolled chamber with a continuous flow of dried air. The morphology of resulting nanofibers varied with humidity, with higher humidity conditions yielding ribbon-like nanofibers and lower humidity (water content below $3 \mathrm{~g} / \mathrm{m}^{3}$ ) yielding cylindrical nanofibers.
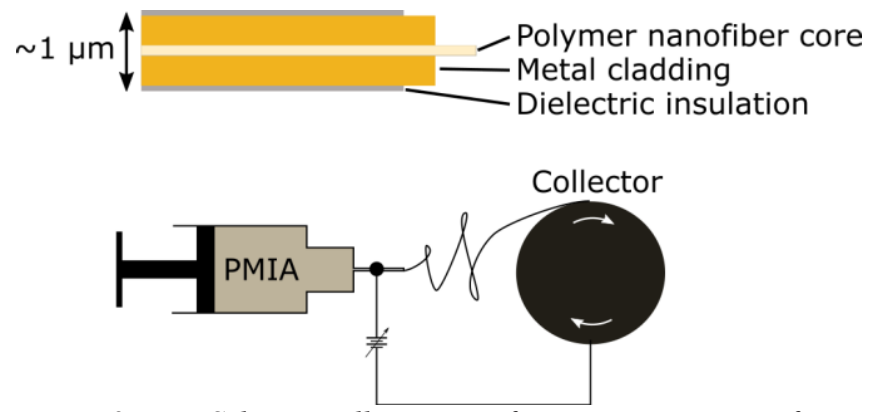

Figure 2: Top: Schematic illustration of target wire geometry for an insulated metal wire of total diameter $\sim 1 \mu \mathrm{m}$. Bottom: Schematic illustration of electrospinning single, continuous nanofiber scaffolds onto a rotating spoked-drum collector.

After electrospinning, nanofiber scaffolds are transferred to rectangular fixtures for subsequent deposition of metal and dielectric layers (Fig. 3). Our process is compatible with a variety of conventional deposition processes, and we have successfully deposited layers via physical vapor deposition (PVD, both sputtering and evaporation), chemical vapor deposition (CVD), and atomic layer deposition (ALD).

Conformal deposition techniques such as CVD can in principle deposit nearly perfectly concentric layers, yet the nucleation of CVD-deposited films is very sensitive to chemical compatibility between the substrate and the deposited material. Therefore, we have predominantly fabricated the metal layer via PVD. Because deposition via PVD is inherently directional, we typically deposit material on one 'side' of the fixture, invert the fixture, and then perform a second deposition on the other side. On average, this process produces wires with substantially conformal layers (Fig. 3). Some nanofibers, however, appear to twist on their axis during deposition, resulting in wires whose nanofiber scaffold is off-center. Nevertheless, for frequencies corresponding to skin depths $\delta$ comparable to or larger than the wire radius, it is expected that there will be negligible electrical impact from wire cross-section geometry that is not perfectly concentric. The asymmetry is expected to modify mechanical properties such as bending stiffness, but we have not observed any impact on the ability to manipulate and twist wires.

An additional concern particular to our choice of PMIA as nanofiber scaffold material is the presence of large amounts of $\mathrm{CaCl}_{2}$ in the nanofiber after the electrospinning process. The $\mathrm{CaCl}_{2}$ improves dissolution of PMIA and enables formation of uniform, aligned nanofibers via electrospinning. As a drawback, $\mathrm{CaCl}_{2}$ is hygroscopic, absorbing ambient moisture subsequent to the spinning process. This water absorption results in local swelling at $\mathrm{CaCl}_{2}$ crystallites, producing protruding features along the length of the wire. The impact of $\mathrm{CaCl}_{2}$ on the long-term reliability of the wires has not been established. It is likely that the $\mathrm{CaCl}_{2}$ could be removed after electrospinning and before metal deposition by, e.g., immersing the nanofibers in water.

\section{WIRE MANIPULATION}

For typical metal wires, conventional manual wire manipulation strategies work well for wire diameters of $\sim 25 \mu \mathrm{m}$ and larger, where the tensile strength is above $0.1 \mathrm{~N}$, the wire can support many grams of mass, and gravity is the strongest ambient force per unit length on the wire. Wire is typically stored on spools, and 
during twisting the wire is pulled off the spools. The rotational motion that results in the twist is generated by moving the spools around each other, while tension is controlled through mechanical means (e.g., by passing the wire under a weighted pulley).
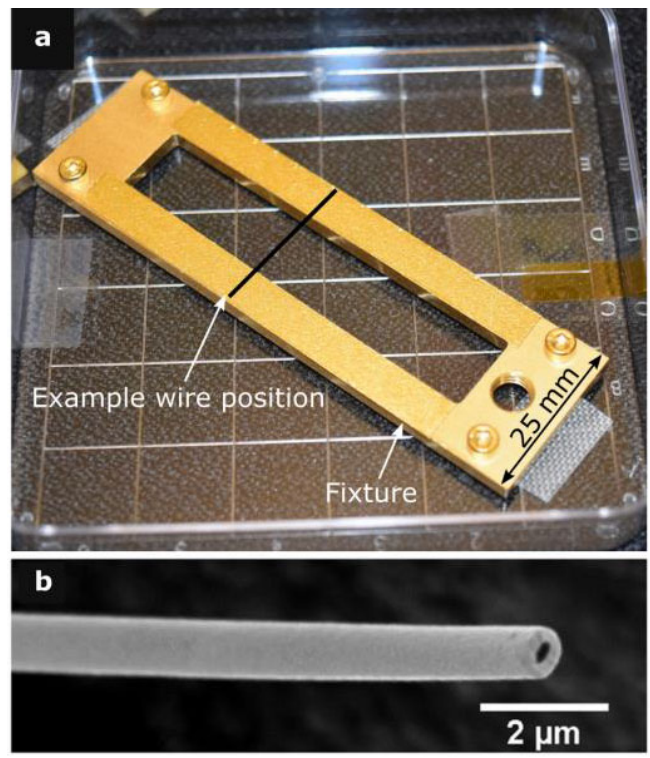

Figure 3: (a), Image of a rectangular fixture containing nine Aucoated nanofibers. Individual wires (not visible at this scale) are oriented as indicated by the black line labeled 'example wire position'. (b), Scanning electron micrograph of a Pt/Pd-coated nanofiber.

In contrast, for wires of diameter $\sim 1 \mu \mathrm{m}$, gravity is several orders of magnitude weaker than other ambient forces such as static electricity and fluid drag from air disturbances, and the tensile strength is less than $300 \mu \mathrm{N}$. Therefore, despite the macroscopic lengths of such wires, their extremely small diameters require that new techniques be developed for manipulation. Our approach to handling and twisting differs from conventional twisting in several respects (Fig. 4). First, to reduce the influence of uncontrolled ambient forces, twisting and bundling of wires is performed while submerged in fluid. Second, tensile force is generated not mechanically but hydrodynamically by controlling fluid flow along the axes of the wires. This is accomplished by placing each wire inside a separate fluid flow channel (with one end extending out of the channel). Third, our wires are stored and inserted into the twisting apparatus in straight individual sections of $\sim 20 \mathrm{~mm}$ length. Fourth, the rotational motion responsible for twisting is generated by an electric motor at the single common attachment of all wires in the bundle. It is worth noting that our approach causes each wire rotate on its axis with the same frequency as the motor, resulting in torsional strain that can lead to torsional buckling and formation of plectonemes if it is not properly mitigated (e.g. via wire tension)[7].

The force required for twisting can be estimated from simple elastostatics of bending a slender rod. For $\sim 1 \mu \mathrm{m}$-diameter copper wires, twisting with a pitch of $\sim 50 \mu \mathrm{m}$ should require $\mu \mathrm{N}$ forces or smaller, depending on the point of application of the force. The force from axial fluid drag can be estimated as Stokes drag via the equation $F \sim 4 \pi \mu l u / \ln (l / a)$, where $\mu$ is the dynamic viscosity of the fluid, $u$ is the velocity of the fluid relative to the wire, $l$ is the wire length over which the drag force is operating, and $a$ is the wire radius. Using water as a fluid, we are able to twist wires into a pitch of $\sim 50 \mu \mathrm{m}$ using a fluid velocity of only $\sim 1 \mathrm{~m} / \mathrm{min}$, which is
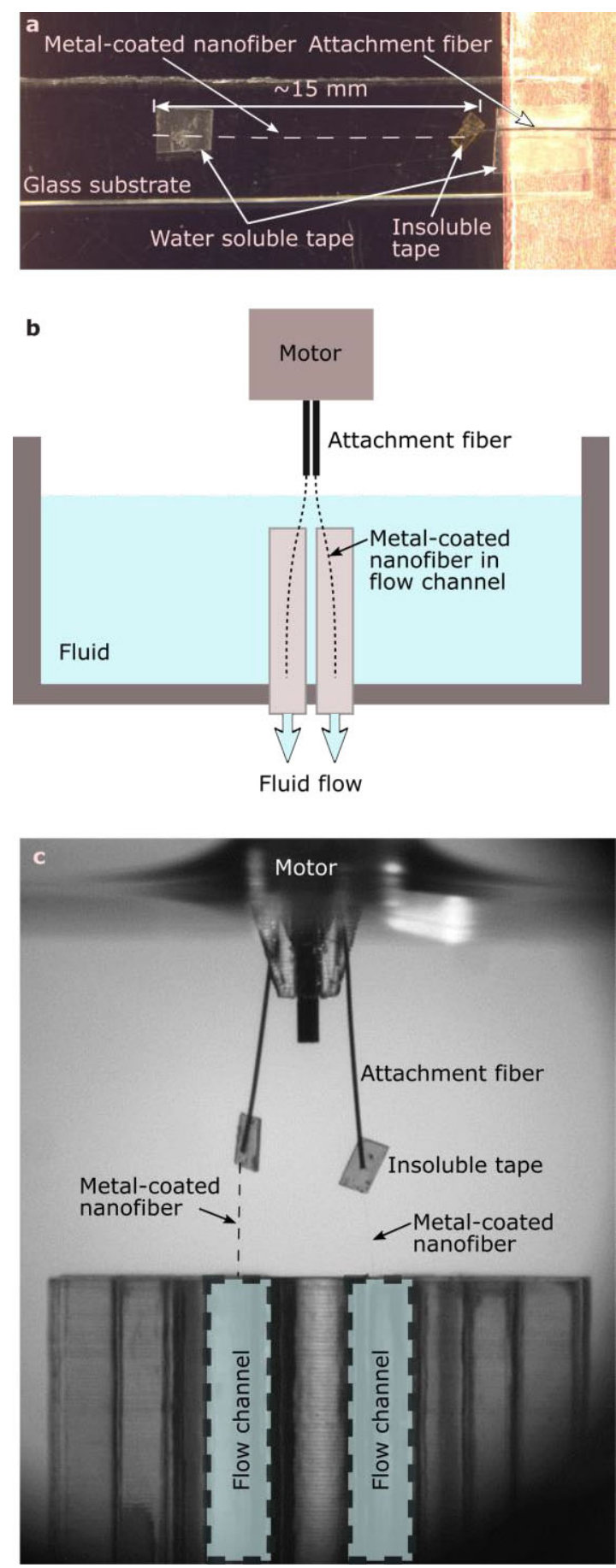

Figure 4. (a), Optical micrograph of a nanofiber (faintly visible; overlaid with dashed line) mounted on glass substrate in preparation for twisting. (b), Schematic diagram of twisting apparatus. (c), Side view image of twisting apparatus during initialization of the system prior to twisting two metal-coated nanofibers. The metal-coated nanofibers ( 1 $\mu$ m diameter) are nearly invisible; dashed line indicates position of the one on the left.

estimated to generate less than $50 \mathrm{nN}$ of force per millimeter of wire. This value is generally consistent with the estimates above given the level of uncertainty around factors such as position of the wire within the flow channel. If needed, larger drag forces can be 
generated by increasing flow rate, decreasing cross-sectional area of the channel, or using a fluid with higher viscosity.

Once each wire is isolated in its own fluid flow channel and has one end attached to the spindle of the electric motor, twisting is accomplished by slowly pulling the motor away from the assembly of fluid flow channels (i.e. in the $+z$ direction) while the motor rotates. The pitch of the twist is primarily determined by the ratio of linear velocity to rotational velocity of the motor, provided that the tension applied to the wires is sufficient to provide the necessary bending force for a given bend radius.

While the twisting process itself is relatively straight forward, it can be challenging to achieve the necessary initial conditions of having several individual metal-coated nanofibers, each isolated in its own fluid flow channel, with one end protruding from the channel and attached to the spindle of a motor. One approach we have developed is to first anchor each metal-coated nanofiber to a separate carrier substrate (e.g. $25 \mathrm{~mm}$ x $5 \mathrm{~mm}$ x $1 \mathrm{~mm}$ glass) using water-soluble tape. We then attach one end of each metal-coated nanofiber to one end of a separate macroscopic fiber (e.g., $\sim 100$ $\mu \mathrm{m}$ diameter monofilament) using permanent adhesive. The carrier substrates are inserted into an assembly of adjacent flow channels, and the free end of the macroscopic fiber is then attached to the motor. Once the assembly is submerged in water, the water-soluble tape dissolves, achieving the desired initial condition.

Using this wire fabrication twisting procedure, we have successfully twisted a variety of bundles of wires (Fig. 5). The number of individual strands is limited by the number of flow channels in the apparatus, but as in conventional litz wire manufacturing, larger bundles can be assembled by heirarchically twisting bundles of twisted bundles (Fig. 5 c-e). Using this approach, we can therefore fabricate litz wire designed for operation in the $\mathrm{GHz}$ regime.

\section{CONCLUSIONS}

Resistive loss due to skin effect increases at high frequency, but conventional approaches to mitigating skin effect do not scale to dimensions that allow use at $\mathrm{GHz}$ frequencies and higher. By developing a novel bottom-up wire fabrication approach as well as a method to tension and twist micron-scale wire, we enable fabrication of high frequency conductors containing the requisite topology to mitigate skin effect at $\mathrm{GHz}$ frequencies.

This work is sponsored by the Air Force Research Laboratory (AFRL) and the Defense Advanced Research Agency (DARPA).

\section{REFERENCES}

[1] G. W. Dahlmann and E. M. Yeatman, "High Q microwave inductors on silicon by surface tension self-assembly," Electron. Lett., vol. 36, no. 20, pp. 1707-1708, Sep. 2000.

[2] G. W. Dahlmann, E. M. Yeatman, P. R. Young, I. D. Robertson, and S. Lucyszyn, "MEMS high Q microwave inductors using solder surface tension self-assembly," in Microwave Symposium Digest, 2001 IEEE MTT-S International, 2001, vol. 1, pp. 329-332.

[3] C. L. Chua, D. K. Fork, K. Van Schuylenbergh, and J.-P. Lu, "High Q RF coils on silicon integrated circuits," 2003, p. 150.

[4] S. Wang, M. A. de Rooij, W. G. Odendaal, J. D. van Wyk, and D. Boroyevich, "Reduction of high-frequency conduction losses using a planar litz structure," IEEE Trans. Power Electron., vol. 20, no. 2, pp. 261-267, Mar. 2005.

[5] Y. Zhao, M. Nandra, C. Yu, and Y. Tai, "Reduction of AC resistance in MEMS intraocular foil coils using microfabricated planar Litz structure," in 2012 th IEEE International Conference on Nano/Micro Engineered and Molecular Systems (NEMS), 2012, pp. 234-237.
[6] C. R. Sullivan, "Optimal choice for number of strands in a litz-wire transformer winding," IEEE Trans. Power Electron., vol. 14, no. 2, pp. 283-291, Mar. 1999.

[7] B. Lautrup, Physics of Continuous Matter, Second Edition: Exotic and Everyday Phenomena in the Macroscopic World. CRC Press, 2011.
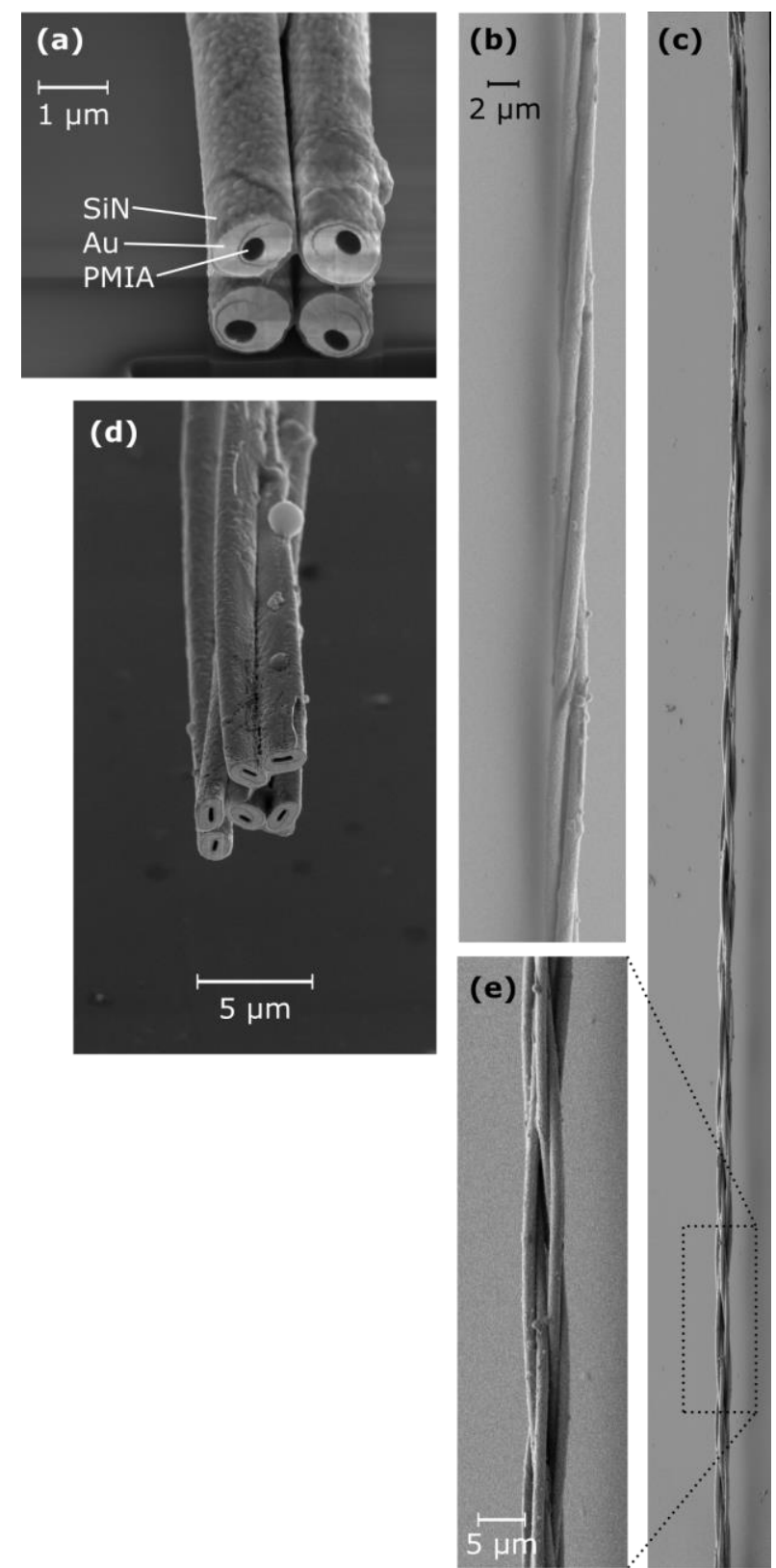

Figure 5. Scanning electron micrographs of fabricated bundles of metal-coated nanofibers. (a), Cross-section of a bundle of four Aucoated PMIA nanofibers insulated with sputtered SiN. (b), Twisted bundle of three Au-coated nanofibers. (c), Heirarchically-twisted bundle of three pairs of Au-coated nanofibers (six total wires). (d), Cross-section of the bundle in (c). The non-circular geometry was the result of excessive humidity during electrospinning. (e),

Higher-resolution image of indicated region of the bundle in (c).

\section{CONTACT}

*Kasey J. Russell, krussell@draper.com 